Volume 1, Issue 1 | Pages 1-8

Received: 17 Mar 2019 | Accepted: 22 Mar 2019 | Published: 29 Mar 2019

\title{
ASPECT BASED SENTIMENT ANALYSIS FOR E-COMMERCE USING CLASSIFICATION TECHNIQUES
}

\author{
ASHWATH K* \& VINOTH J \\ Research Scholar, Department of Industrial and Systems Engineering, Bharath Niketan Engineering college, \\ Theni, Tamil Nadu, India \\ *Corresponding Author: Ashwath K, B.Tech(civil), M.Tech, Department of Industrial and Systems Engineering, \\ Bharath Niketan Engineering college, Theni, Tamil Nadu, India
}

\section{$\underline{\text { ABSTRACT }}$}

Tremendous accumulations of shopper audits for items are currently accessible on the Web. These audits contain rich stubborn data on different items. They have turned into an important asset to encourage shoppers in understanding the items preceding settling on buying choices, and bolster makers in fathoming purchaser suppositions to successfully enhance the item contributions. In any case, such audits are frequently sloppy, prompting trouble in data route and information securing. It is wasteful for clients to accumulate general suppositions on an item by perusing all the shopper audits and physically investigating assessments on each survey. In this undertaking, we can actualize item surveys rating from item audits, which intend to naturally distinguish critical item perspectives from online buyer surveys. The imperative viewpoints are recognized by two perceptions: the vital parts of an item are typically remarked by an expansive number of shoppers; and buyers' conclusions on the essential angles significantly impact their general sentiments on the item. Specifically, given customer audits of an item, we initially recognize the item angles by marking the surveys and decide buyers' feelings on these perspectives by means of a slant classifier. The Proposed research can be execute SVM and Naive Bayes arrangement to recognize the supposition words by at the same time thinking about the surveys gathering and the impact of purchasers' assessments given to every perspective on their general sentiments. The exploratory outcomes on prevalent portable item surveys show the adequacy of our approach. We additionally apply the survey positioning outcomes to the utilization of assessment order, and enhance the execution essentially.

KEYWORDS: shoppers, surveys, impact of purchasers, utilization of assessment order

\section{INTRODUCTION}

The most vital goal of Aspect Based Sentiment Analysis is to distinguish the parts of the given target substances and notion communicated for every perspective. The goals of Aspect Based Sentiment Analysis should be possible through the accompanying errands. The main undertaking is 
the extraction of viewpoint terms and gathering angle terms into perspective classifications. The second undertaking is about recognizable proof of extremity of the perspective terms and extremity of the angle classes of each sentence. The above errands are partitioned into four sub undertakings to be specific: Aspect Term Extraction (ATE), Aspect Term Polarity (ATP), Aspect Category Detection (ACD) and Aspect Category Polarity (ACP). Crafted by first sub - errand Aspect Term Extraction (ATE) is otherwise called data extraction undertaking is to recognize all the perspective terms given in each audit sentence. There can be numerous angles, in a sentence and each viewpoint should be extricated. The perspective in the angle terms of the sentence can be communicated by a thing, verb, modifier and descriptive word. The second sub - assignment is viewpoint term extremity is that, inside a sentence for a given arrangement of perspective terms, the errand is to decide the extremity of every angle term: positive, negative, impartial or strife (i.e., both positive and negative). Here in the ID of Aspect term extremity diverse highlights like Word N - grams, Polarity of neighboring descriptive words, Neighboring POS labels and Parse conditions and relations have been generally utilized by specialists. The third sub - errand is Aspect Category Detection, in which the assignment is to recognize the larger part of classifications that are talked about in each sentence. Asp ect classifications are generally hard to discover than the viewpoint terms as characterized in Aspect Term Extraction, and now and again they don't happen as terms in the sentence. Viewpoint classification recognition depends on an arrangement of twofold Maximum Entropy classifiers. A ultimate conclusion is only computed from choices of different individual classifiers. The last sub errand is Aspect Category Polarity is which it takes the data from the past undertaking (Aspect Category Detection) to decide the extremity of every angle classification talked about in audit sentence. The feeling of viewpoint class is processed by figuring the separation between $\mathrm{n}$ - gram and the relating angle.

\section{CONTRibutions}

After the critical review of the related field, gaps are recognized which results in the following contribution to the body of knowledge.

- A fully developed aspect level sentiment analysis for the online products.

- In determining the relationship between the aspects of the reviews.

- A critical comparison between the evaluated results of the different models (Neural Network, SVM).

- The configuration manual utilized in the development and implementation of the project.

In order to answer the proposed research question, the project follows a modified KDD 
methodology. The methodology stages are modified according to the need of the project and it fits best for this research.

\section{FRAMEWORK ARCHITECTURE}

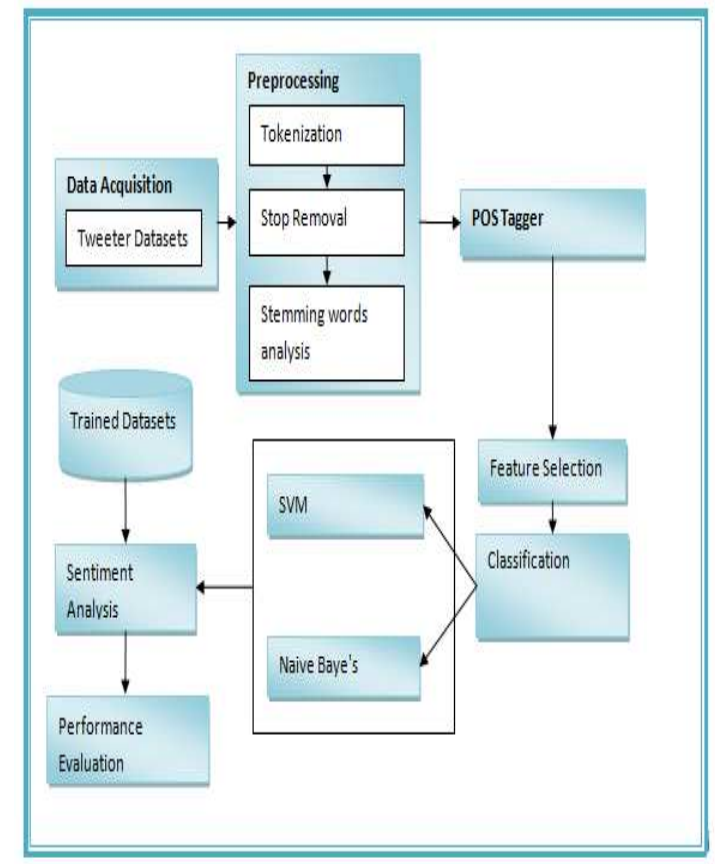

\section{IMPLEMENTATION STEPS}

\section{UPLOAD DATASETS}

Feeling is individual perspective around a question while mining is the extraction of learning from actualities or crude information. In this way, in another word it is a procedure which recognizes canny data from information open on web. The general population who express their feeling on web has significantly step by step. They can express their supposition relatively in view of User Generated Content audit locales, gatherings, dialogs gatherings, online journals, items and so on. In view of above site, we can gather client audits about mobiles.

\section{Preprocessing}

In this module, we can kill stop words and stemming words in view of POS tagger. In corpus etymology, grammatical feature labeling (POS labeling or POST), additionally called linguistic labeling or word-classification disambiguation, is the way toward increasing a word in a content (corpus) as comparing to a specific grammatical feature, in view of the two its definition and its unique situation-i.e., its association with adjoining and related words in an expression, sentence, or passage. In registering, stop words will be words which are sifted through previously or subsequent 
to handling of characteristic dialect information (content). In spite of the fact that stop words ordinarily allude to the most widely recognized words in a dialect, there is no single all inclusive rundown of stop words utilized by all common dialect preparing devices, and for sure not all instruments even utilize such a rundown. In computational phonetics, a stem is the piece of the word that never shows signs of change notwithstanding when morphologically curved, and a lemma is the base type of the word. Stemming words are additionally expelled from client surveys. At that point execute POS tagger that peruses message in some dialect and relegates parts of discourse to each word (and other token, for example, thing, verb, descriptive word, and so on., albeit for the most part computational applications utilize all the more fine-grained POS labels like 'thing plural'.

\section{ASPeCt Detection}

It has been watched that in surveys, a constrained arrangement of words is utilized substantially more regularly than whatever is left of the vocabulary. These successive words (typically just single things and compound things are considered) are probably going to be perspectives. This direct technique ends up being very intense, a reality exhibited by the critical number of methodologies utilizing this strategy for angle location. Clear deficiencies are the way that not every single regular thing are really alluding to perspectives. A few things in purchaser audits, for example, 'Screen' or 'show', are simply every now and again utilized. The viewpoint discovery strategy depicted in just thinks about single things and compound things as conceivable angles. Initially, the recurrence of every mix of things is recovered. For this, the things don't need to be by each other, they should simply show up in a similar sentence. This discovers angles like 'screen measure' when it is expressed as 'size of the screen'.

\section{SVM}

This suggest the item in view of arrangement by utilizing SVM approach. The info space is arranged into a high dimensional component space. At that point, the hyper plane that adventures the edge of partition between classes is built. The focuses that lie nearest to the choice surface are called bolster vectors straightforwardly includes its area. At the point when the classes are nondistinguishable, the ideal hyper plane is the one that limits the likelihood of grouping blunder. At first information survey is detailed in include vectors. At that point these element vectors mapped with the assistance of piece work in the element space. Lastly division is figured in the element space to isolate out the classes for preparing information. A worldwide hyper plane is required by the SVM keeping in mind the end goal to separate both the program of cases in preparing set and maintain a strategic distance from over fitting. This marvel of SVM is higher in contrast with other machine learning procedures which depend on computerized reasoning. Here the critical element for the order 
is the width of the vessels. With the assistance of SVM classifier we can without much of a stretch separate out the vessels into supply routes and veins. The SVMs exhibit different alluring highlights, for example, great speculation capacity contrasted with different classifiers. To be sure, there are generally few free parameters to change and it isn't required to discover the design tentatively. The SVMs calculation isolates the classes of info designs with the maximal edge hyper plane.

This hyper plane is constructed as:

$$
f(x)=\langle w, x\rangle+b
$$

Where $\mathrm{x}$ is the feature vector, $\mathrm{w}$ is the vector that is perpendicular to the hyper plane and $b\|w\|^{-1}$ specifies the offset from the beginning of the coordinate system. To benefit from non-linear decision boundaries the separation is performed in a feature space $\mathrm{F}$, which is introduced by a nonlinear mapping $\varphi$ the input patterns. This mapping is defined as follows:

$$
\left\langle\varphi\left(x_{1}\right), \varphi\left(x_{2}\right)\right\rangle=K\left(x_{1}, x_{2}\right) \forall\left(x_{1}, x_{2}\right) \in X
$$

for some kernel function $\mathrm{K}(\cdot, \cdot)$. The kernel function represents the non-linear transformation of the original feature space into the F. Finally recommend books which are positive opinion words.

\section{NAÏVE BAYES}

Naïve Bayes Method It is a probabilistic classifier and is mainly used when the size of the training set is less. In machine learning it is in family of sample probabilistic classifier based on Bayes theorem. The conditional probability that an event $\mathrm{X}$ occurs given the evidence $\mathrm{Y}$ is determined by Bayes rule by the (1).

$$
P(X / Y)=P(X) P(Y / X) / P(Y)
$$

So for finding the sentiment the equation is transformed into the below (2).

$\boldsymbol{P}($ Sentiment/Sentence $)=\boldsymbol{P}($ Sentiment $) \boldsymbol{P}($ Sentence $/$ Sentiment $) / P($ Sentence $)$

$P$ (sentence/sentiment) is calculated as the product of P (token /sentiment) [5], which is formulated by the (3).

Count(Thistokeninclass)+1/Count(Alltokensinclass)+Count(Alltokens)

Here 1 and count of all tokens is called add one or Laplace smoothing

\section{RESULTS AND DISCUSSIONS}

At last, a framework that can identify slant and anticipate their veracity and possibly affect is without a doubt an exceptionally profitable and valuable instrument. In any case, sometimes the clients of the framework should need to hose the impacts of assessment examination, particularly ones that are anticipated to be false and impactful.

The order precision rates for the datasets were estimated. For instance, in the arrangement 
issue with two-classes, positive and negative, a single expectation has four probability. The True Positive rate (TP) and True Negative rate (TN) are right arrangements. A False Positive (FP) happens when the result is erroneously anticipated as positive when it is really negative. A False Negative (FN) happens when the result is erroneously anticipated as negative when it is really positive.

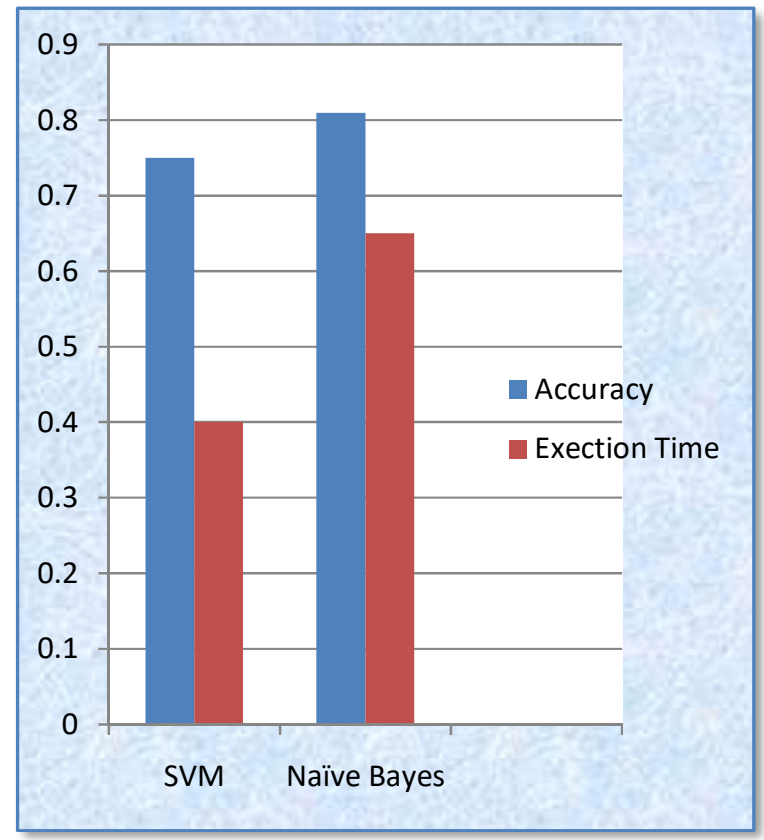

\begin{tabular}{|l|c|}
\hline \multicolumn{1}{|c|}{ METHODS } & ACCURACY \\
\hline Naive Bayes & $81.30 \%$ \\
\hline SVM & $76.22 \%$ \\
\hline
\end{tabular}

\section{CONCLUSIONS}

Different notion examination techniques and its diverse levels of breaking down suppositions have been considered in this paper. Our definitive point is to think of Sentiment Anal y sister which will effectively arrange different audits. Machine learning techniques like SVM, NB, strategies were talked about here in a word, alongside some other intriguing techniques that can enhance the investigation procedure in either way. Semantic examination of the content is of incredible thought. Research work is done for better investigation strategies around there, including the semantics by considering $\mathrm{n}$ - gram assessment rather than word by word examination. We have likewise gone over some different strategies like control based and vocabulary based techniques. In the realm of Internet lion's share of individuals rely upon long range interpersonal communication destinations to get their esteemed data, breaking down the audits from these online journals will yield a superior comprehension and help in their choice - making. 


\section{FUTURE ENHANCEMENT}

Results got for the positioning of perspectives are likewise promising In future work, endeavors would be done to make a few improvements in this method such that it can distinguish the rehashed surveys and characterize those audits just once. It would manage the sentences contain relative conditions like as well as and the sentences contain provisos not one or the other nor, eitheror and so forth.

\section{REFERENCES}

1. G. Adomavicius, and A. Tuzhilin, "Convolutional Neural Networks for Sentence Classification," IEEE Transactions on Knowledge and Data Engineering, pp. 734-749, Jun. 2005.

2. Y. Koren, “Locations Recommendation Based on Check-in Data from Location-based Social Network," KDD'08, 2008.

3. Y. Koren, "Friendship and Mobility: User Movement In Location-Based Social Networks,” KDD'09, pp. 447-456, 2009.

4. J. Wang, A. P. d. Vries, and M. J. T. Reinders, "Service Objective Evaluation via Exploring Social Users' Rating Behaviors, "SIGIR'06, 2006.

5. N. N. Liu, M. Zhao, and Q. Yang, "Aspect Specific Sentiment Analysis using Hierarchical Deep Learning," CIKM'09, pp. 759-766, 2009.

6. Q. Liu, E. Chen, H. Xiong, C. Ding, and J. Chen, "Spatiotemporal Sequential Influence Modeling for Location Recommendations: A Gravity-based Approach,” IEEE Transactions on Systems, Man, and Cybernetics- Part B, pp. 218-233, Feb.2012.

7. Y. Chen, and J. Canny, "Recommending ephemeral items at web scale,” SIGIR, pp. 1013-1022, 2011.

8. M. Harvey, M. J. Carman, I. Ruthven, and F. Crestani, "Identifying Noun Product Features that Imply Opinions," CIKM'11, pp. 699-708, 2011.

9. M. Jamali, and M. Ester, “Clustering for Simultaneous Extraction of Aspects and Features from Reviews," ACM RecSys, 2010.

10. Duyu Tang, and A. Tuzhilin, "Convolutional Neural Networks for Sentence Classification," IEEE Transactions on Knowledge and Data Engineering, pp. 734-749, Jun. 2005.

11. Anindya Ghose, "Estimating the Helpfulness and Economic Impact of Product Reviews: Mining Text and Reviewer Characteristics," World Wide Web, pp. 285-295, 2010.

12. Eduard C. Dragut, “Polarity Consistency Checking for Domain Independent Sentiment Dictionaries,” KDD’08, 2015 .

13. P. L. Ramteke, "Rating Prediction based on Social Sentiment from Textual Reviews," KDD'09, pp. 447-456, 2017.

14. H Nguyen, A. P. d. Vries, and M. J. T. Reinders, "A Deep Neural Architecture for Sentence-level Sentiment Classification in Twitter Social Networking,” SIGIR'06, 2017.

15. N. N. Liu, M. Zhao, and Q. Yang, "Dual Sentiment Analysis: Considering Two Sides of One Review," CIKM'09, pp. 759-766, 2009.

16. K. Lavanya, "TASC: Topic-Adaptive Sentiment Classification on Dynamic Tweets," IEEE Transactions on Systems, Man, and Cybernetics- Part B, pp. 218-233, Feb.2016.

17. Kushal Dave, "Mining the Peanut Gallery: Opinion Extraction and Semantic Classification of Product Reviews" SIGIR, pp. 1013-1022, 2011.

18. Xiaowen Ding, Bing Liu, “A Holistic Lexicon-Based Approach to Opinion Mining,” CIKM'11, pp. 699-708, 2008.

19. Nal Kalchbrenner Edward Grefenstette, "A Convolutional Neural Network for Modelling Sentences," ACM RecSys, 2010.

20. Tony Mullen and Nigel Collier, "Sentiment analysis using support vector machines with diverse information sources" SIGIR, pp. 1013-1022, 2011. 
21. O. T"̈ackström and R. McDonald. Semi-supervised latent variable models for sentence-level sentiment analysis. In ACL, pages 569-574, 2011.

22. Duyu Tang, Furu Wei, "Learning Sentiment-Specific Word Embedding for Twitter Sentiment Classification," CIKM'11, pp. 699-708, 2008.

23. J. Duchi, E. Hazan, and Y. Singer. Adaptive subgradient methods for online learning and stochastic optimization. JMLR, 12:2121-2159, 2011.

24. J. McAuley, R. Pandey, and J. Leskovec. Inferring networks of substitutable and complementary products. In SIGKDD, pages 785-794, 2015. 